\title{
Relaxation in Time-Dependent Current-Density-Functional Theory
}

\author{
Roberto D'Agosta and Giovanni Vignale \\ Department of Physics and Astronomy, University of Missouri, Columbia, Missouri 65211, USA
}

(Received 6 August 2005; published 11 January 2006)

\begin{abstract}
We apply the time-dependent current-density-functional theory to the study of the relaxation of a closed many-electron system evolving from a nonequilibrium initial state. We show that the self-consistent unitary time evolution generated by the exchange-correlation vector potential irreversibly drives the system to equilibrium. We also show that the energy dissipated in the Kohn-Sham system, i.e., the noninteracting system whose particle and current densities coincide with those of the physical system under study, is related to the entropy production in the real system.
\end{abstract}

The irreversible time evolution of a many-electron system which is initially prepared in a nonequilibrium state is the subject of strong research interest [1]. The system might be, for example, a large molecule [2] or the electron liquid in a quantum well, and the initial nonequilibrium state might be created by laser excitation or by the application and subsequent removal of an electric field [3]. From experience, we know that, even if the system is closed and isolated, i.e., no exchanges of particles and energy are allowed, the interaction between the particles drives the system towards the state of maximum entropy, i.e., the equilibrium state. When this happens, we say that the system has relaxed [4]. At each stage of the relaxation process, the system has a certain amount of "mechanical energy" which could, in principle, be channeled into work. One of the tasks of theory is to develop efficient tools - as an alternative to the solution of the many-body Schrödinger equation - to calculate the time evolution of the mechanical energy. One would like to do this starting from first principles yet without knowing the exact state of the many-body system as a function of time.

Various formulations of the problem of relaxation of a quantum system have been proposed over the years. Most of these formulations couple the system of interest to some kind of external environment - the so-called thermal bath [5]. When the information about the microscopic state of the thermal bath is discarded, this approach leads to an effective dynamics of the system that is no longer unitary. The main weakness of this approach lies in the fact that the thermal bath and its coupling to the system must be modeled in an essentially ad hoc manner. In this Letter, we will describe an alternative approach, based on the timedependent current-density-functional theory (TDCDFT) [6-10], which allows a first-principle treatment of relaxation in terms of a self-consistent unitary time evolution [11].

We assume that the external potential acting on the system does not depend on time and that the system is closed and isolated. We thus consider the time evolution of a many-electron system described by the Hamiltonian

$$
\hat{H}=\sum_{i} \frac{\hat{p}_{i}^{2}}{2 m}+\hat{W}+\int d r V(r) \hat{n}(r),
$$

where $\hat{n}(r)$ is the density operator, $V(r)$ is the external potential, and $\hat{W}$ describes the electron-electron interaction (here and in the following, $\hbar=1$ ). A possible way to generate the initial nonequilibrium state $\left|\psi_{i}\right\rangle$ is by applying an external potential at $t=-\infty$ and allowing the system to relax in the presence of this potential. The external potential is then switched off at the initial time $t=0$, and the system evolves henceforth according to the Schrödinger equation $i \partial_{t}|\Psi(t)\rangle=\hat{H}|\Psi(t)\rangle$. Because of the presence of the electron-electron interaction, the system will relax to an equilibrium state of $\hat{H}$. Because the system is closed and isolated during its time evolution, the energy is conserved and equal to its initial value $E_{i}=\left\langle\psi_{i}|\hat{H}| \psi_{i}\right\rangle$. This implies that the final equilibrium state will not be the ground state of $\hat{H}$ but the most probable state with total energy $E_{i}$. In this final state, the entropy, as well as the temperature, will have finite values. Our task is to calculate the time evolution of the mechanical energy between the initial and the final states of this evolution.

The central tenet of TDCDFT is that the correct time evolution of the particle and current densities of the manyelectron system can be obtained from the time evolution of a noninteracting reference system, known as the KohnSham (KS) system, described by the state $\left|\Psi_{\mathrm{KS}}(t)\right\rangle$. This state evolves from an initial state $\left|\Psi_{\mathrm{KS}, i}\right\rangle$-constructed to give the initial density and energy of the system-according to the equation

$$
i \partial_{t}\left|\Psi_{\mathrm{KS}}(t)\right\rangle=\hat{H}_{\mathrm{KS}}(t)\left|\Psi_{\mathrm{KS}}(t)\right\rangle,
$$

where the KS Hamiltonian is

$$
\begin{aligned}
\hat{H}_{\mathrm{KS}}(t)= & \sum_{i} \frac{1}{2 m}\left[\hat{p}_{i}+e A_{x c}\left(\hat{r}_{i}, t\right)\right]^{2}+\int d r \hat{n}(r) V_{h x c}(r, t) \\
& +\int d r \hat{n}(r) V(r) .
\end{aligned}
$$

$V_{h x c}(r, t)$ is an effective scalar potential that includes the 
Hartree potential $(h)$ as well as the ground-state exchangecorrelation $(x c)$ potential of static density-functional theory (DFT) evaluated at the instantaneous density $n(r, t)$. $A_{x c}(r, t)$ is the $x c$ vector potential, which is a functional of the density and of the time-dependent current density $j(r, t)$ and whose role is to enforce the correct time evolution of the current. The time dependence of $\hat{H}_{\mathrm{KS}}$ arises from its being a functional of $n$ and $j$. In practice, both $V_{h x c}(r, t)$ and $A_{x c}(r, t)$ need to be approximated, but these approximations are nonempirical, in the sense that they are based on exact physical constraints. The simplest and most popular approximation is the local density approximation (LDA), which expresses the $x c$ potential as a functional of the density in the following manner:

$$
V_{h x c}(r, t)=\int d r^{\prime} \frac{e^{2}}{\left|r-r^{\prime}\right|} n\left(r^{\prime}, t\right)+\mu_{x c}(n(r, t)),
$$

where $\mu_{x c}(n)=d \epsilon_{x c}(n) / d n$ and $\epsilon_{x c}(n)$ is the $x c$ energy density of a uniform electron gas [10]. As for $A_{x c}$, a quasilocal approximation in terms of the current density was derived by Vignale and Kohn [12] and can be cast in the form [13]

$$
e \frac{d}{d t} A_{x c, k}(r, t)=\frac{1}{n(r, t)} \nabla_{i} \sigma_{x c}^{i k}(r, t),
$$

where $i, k$ denote Cartesian components and the stress tensor $\sigma_{x c}$ is given by

$$
\begin{aligned}
\sigma_{x c}^{i k}(r, t)= & \zeta(r, t) \delta_{i k} \nabla \cdot v+\eta(r, t) \\
& \times\left(\nabla_{i} v_{k}+\nabla_{k} v_{i}-\frac{2}{3} \delta_{i k} \nabla \cdot v\right),
\end{aligned}
$$

where $v(r, t)=j(r, t) / n(r, t)$ is the velocity, and we have, for simplicity, neglected retardation effects in the relation between $\sigma_{x c}$ and the velocity. The quantities $\eta$ and $\zeta$ are the viscoelastic constants of the homogeneous electron liquid and can be calculated from the linear response functions of the latter [10], evaluated at the instantaneous density $n(r, t)$. We want to point out that Eqs. (2)-(6) define a self-consistent unitary evolution which is designed to approximate the true density and current of the system. It is the dependence of the $x c$ vector potential on the velocity that breaks the time-reversal invariance of the KS Hamiltonian and allows for the possibility of irreversible relaxation.

We now define the key quantity of this Letter, the "Kohn-Sham energy"

$$
\begin{aligned}
E(t) \equiv & \left\langle\Psi_{\mathrm{KS}}(t)\left|\hat{T}_{A_{x c}}+\hat{V}\right| \Psi_{\mathrm{KS}}(t)\right\rangle+E_{h x c}[n(t)] \\
= & \left\langle\Psi_{\mathrm{KS}}(t)\left|\hat{H}_{\mathrm{KS}}(t)\right| \Psi_{\mathrm{KS}}(t)\right\rangle \\
& -\int d r V_{h x c}(r, t) n(r, t)+E_{h x c}[n(t)],
\end{aligned}
$$

where $\hat{T}_{A_{x c}}=\sum_{i}\left(\hat{p}_{i}+e A_{x c}\left(\hat{r}_{i}, t\right)\right)^{2} / 2 m$ is the kinetic energy operator, and $E_{h x c}$ is the Hartree $+x c$ energy functional of the ground-state DFT. $E_{h x c}$ and $V_{h x c}$ are related by the identity $V_{h x c}=\delta E_{h x c}(n) / \delta n$. In static DFT, $E(t)$ is just the familiar expression from which the ground-state energy is calculated. In TDCDFT, $E(t)$ is not the true energy of the interacting many-particle system, but, as we will show below, it decreases monotonically with time going from $E_{i}$ at $t=0$ to some final value $E_{f} \geq E_{0}$ at $t=+\infty, E_{0}$ being the true ground-state energy of the system.

To prove this point, we observe that a straightforward calculation shows that (we used $\delta \hat{H}_{\mathrm{KS}} / \delta A_{x c}=\hat{j}$ )

$$
\frac{d E}{d t}=\int d r j(r, t) \cdot \frac{d A_{x c}}{d t} .
$$

Equation (8) immediately allows us to identify $d E / d t$ as the work done on the system by the self-consistent $x c$ vector potential. By putting Eq. (5) in Eq. (8), we get

$$
\frac{d E}{d t}=-2 \int d r \eta \operatorname{Tr}\left(\omega-\frac{1}{3} \operatorname{Tr} \omega\right)^{2}-\int d r \zeta(\operatorname{Tr} \omega)^{2},
$$

where $\omega_{i j}=\left(\nabla_{i} v_{j}+\nabla_{j} v_{i}\right) / 2$. The nonpositivity of $d E(t) / d t$ follows from the fact that the viscosity constants $\eta$ and $\zeta$ are positive [10].

Next we prove that $E(t) \rightarrow E_{f} \geq E_{0}$ for $t \rightarrow \infty$. We begin by observing that, at a fixed time $t$ [14],

$$
\begin{aligned}
E(t) & \geq \min _{\Psi \rightarrow n(t)}\left\langle\Psi(t)\left|\hat{T}_{A_{x c}(t)}+\hat{V}\right| \Psi(t)\right\rangle+E_{h x c}[n(t)] \\
& \geq E_{0}\left[A_{x c}(t), V\right]+E_{h x c}[n(t)]-E_{h x c}\left[n(t), A_{x c}(t)\right],
\end{aligned}
$$

where $E_{0}[A, V]$ is the instantaneous ground-state energy of the system, in the presence of static potentials $V$ and $A$. $E_{h x c}[n, A]$ is the Hartree $+x c$ energy functional of the system in the presence of a static vector potential $A(r)$. Now it is known [15] that, in LDA and up to second order in $B(r) \equiv \nabla \times A(r)$, one has $E_{h x c}[n]-E_{h x c}[n, A]=$ $\int \operatorname{drc}(n(r)) B^{2}(r)>0$, because $c(n)$ (equals one-half the difference between the orbital magnetic susceptibilities of the interacting and noninteracting homogeneous electron liquid [15]) is a positive quantity. Assuming that this inequality is generally true [16], we can then neglect the last two terms on the right-hand side of Eq. (10) to arrive at $E(t) \geq E_{0}\left[A_{x c}(t), V\right]$. We now observe that, because the electron-electron Coulomb interaction is a positive operator, the ground-state energy of an interacting manyparticle system is always greater than the ground-state energy of the same system where electron-electron interaction is turned off. We then conclude that $E_{0}\left[A_{x c}, V\right] \geq$ $E_{0}^{\mathrm{ni}}\left[A_{x c}, V\right]$, where $E_{0}^{\mathrm{ni}}$ is the instantaneous ground-state energy of the noninteracting system. On the other hand, the ground-state energy of a noninteracting fermion system is always greater than the ground-state energy $E_{0, b}^{\mathrm{ni}}$ of a noninteracting boson system in the same external potentials. We then have

$$
E(t) \geq E_{0}^{n i}\left[A_{x c}, V\right] \geq E_{0, b}^{n i}\left[A_{x c}, V\right] \geq E_{0, b}^{n i}\left[A_{x c}=0, V\right],
$$

where we have used in the last step the diamagnetic inequality, which states that the ground-state energy of a spinless boson system in the presence of a vector potential is always greater than the ground-state energy of the 
same system without the vector potential [17]. Because $E_{0, b}^{n i}\left[A_{x c}=0, V\right]$ is a time-independent quantity, we have arrived at our conclusion that $E(t)$ is bounded. Equations (9) and (11) allow us to conclude that the limit $t \rightarrow \infty$ of $E(t)$ is finite and that $\lim _{t \rightarrow \infty} d E / d t=0$. From Eq. (9) and the requirement that $j$ vanishes at infinity, we deduce that we can have $d E / d t=0$ if and only if $\nabla \cdot v=0$ and $\nabla \times v=0$, which imply $v=0$ everywhere (we consider only simply connected geometries). In turn, this implies that the current $j$ is zero everywhere, and, therefore, through the continuity equation, the density approaches a stationary limit $n_{f}(r)$. This can happen only if the asymptotic state is one of the eigenstates of the KS Hamiltonian with density $n_{f}(r)$. In the following, we will disregard the unlikely cases when the system gets stuck in some excited state [18], and we will assume $n_{f}(r)=n_{0}(r)$, where $n_{0}(r)$ is the ground-state density. The time evolution of Eq. (2) will then drive the KS system to the ground state of the corresponding KS Hamiltonian.

To demonstrate the above ideas in a concrete example, we have computed numerically the time evolution of the electronic dipole moment $d(t)=\int d z z n(z, t)$ [Fig. 1(a)] and the energy $E(t)-E_{0}$ [Fig. 1(b)] in a one-dimensional quantum well, such that the electrons are confined in the $z$ direction and yet are free to move in the $x-y$ plane [19]. The single-particle states for this system are of the form $\psi_{n}(z) e^{i k \cdot r}$, where $k$ and $r$ are vectors in the $x-y$ plane. The system is initially prepared in a state that corresponds to the KS ground state in the presence of a uniform electric field $\mathcal{E}=0.01 \mathrm{mV} / \mathrm{nm}$ in the $z$ direction. This state is homogeneous in the $x-y$ plane, and this property is maintained throughout the subsequent time evolution. This means that all the electrons share the same state of motion in the $z$ direction - a time-dependent state that can be written as a linear superposition of the lowest-lying stationary states $\psi_{n}(z)$ (typically the two lowest ones suffice). The $x c$ vector potential $A_{x c}$ has been approximated in the

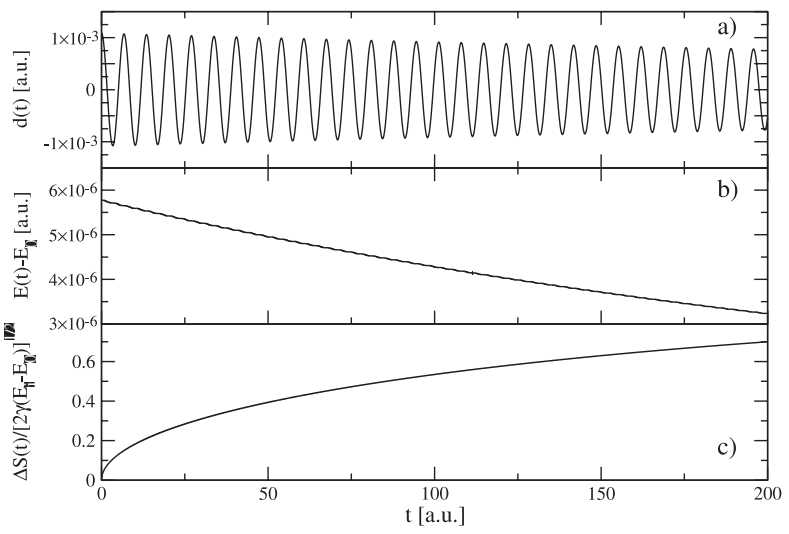

FIG. 1. (a) Plot of the dipole moment $d(t)$ for the electrons in the quantum well as described in the text. (b) Plot of the KohnSham energy $E(t)-E_{0}$. (c) Plot of $\Delta S(t) / \sqrt{2 \gamma\left(E_{i}-E_{0}\right)}$ as given by Eqs. (12) and (15). A fit of $E(t)$ using the data in (b) gives $\Gamma=0.0034$ a.u. form of Eq. (5) [20]. We see from Fig. 1 that the condition $d E / d t \leq 0$ is verified at all times. Moreover, the energy $E(t)$ is well approximated by the analytic form

$$
E(t)-E_{0}=\left(E_{i}-E_{0}\right) e^{-\Gamma t},
$$

where the parameter $\Gamma$ can be estimated from the slope of the curve in Fig. 1.

We now wish to argue that $E(t)-E_{0}$ is the maximum work that can be extracted from the system at a given time and that its time derivative is related to the rate of entropy production [21]. To do this, let us take a closer look to the quantum well (see Fig. 2). In the 3D system, we separate two different sets of degrees of freedom: the motion in the $z$ direction confined in the quantum well and the free motion in the $x-y$ plane. These two sets of degrees of freedom can be studied as two coupled "subsystems" (see Fig. 2). The coupling is induced by the electron-electron Coulomb interaction: No empirical modeling is needed. As the macroscopic state of motion in the $z$ direction evolves with time, energy is transferred from this motion into low-lying excitations of the two-dimensional electron gas (2DEG) in the $x-y$ plane. These excitations are, in the simplest case, double electron-hole pairs with zero total momentum.

We start from an initial state in which the 2DEG is in the ground state but all the electrons occupy an excited state of motion in the $z$ direction. At the end of the relaxation, all the electrons are in lowest energy state in the $z$ direction, but the 2DEG is in an excited state. In the process, we have transferred energy from a macroscopic state to the many degrees of freedom of the 2DEG. The total energy is conserved and given by $E_{i}$. On the other hand, the infinite-time limit of $E(t)$ is the ground-state energy of the system $E_{0}$, as one can see by noting that the final Kohn-Sham Hamiltonian, according to Eq. (3), coincides

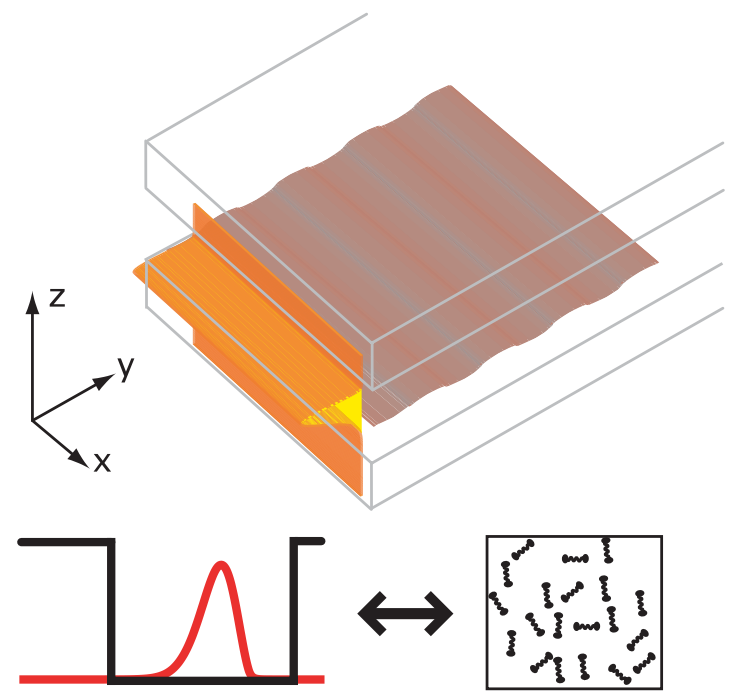

FIG. 2 (color online). The motion in the $z$ direction is coupled through the Coulomb interaction to a 2DEG in the $x-y$ plane. The energy is lost to the motion in the $z$ direction at a rate given by $d E / d t$. 
with the Kohn-Sham Hamiltonian of static DFT which, by definition, yields the correct ground-state energy. Since the electrons are finally at rest in the $z$ direction, the difference $E_{i}-E_{0}$ must be entirely ascribed to the excitation of the 2DEG; i.e., $E_{i}-E_{0}$ is the total heat $Q$, transferred from the macroscopic motion in the $z$ direction to the many degrees of freedom of the 2DEG. Denoting by $T_{i}$ and $T_{f}$ the initial and final temperatures of the $2 \mathrm{DEG}$, respectively, we have

$$
Q=\int_{T_{i}}^{T_{f}} d T C_{V}(T)
$$

where $C_{V}(T)$ is the heat capacity of the 2DEG. At low temperature, we have $C_{V}(T)=\gamma T$, with $\gamma=$ $\pi \mathcal{A} m^{*} k_{B} / 3$, where $\mathcal{A}$ is the area occupied by the gas in the $x-y$ plane and $m^{*}$ is the particle mass renormalized by electron-electron interactions. Because in our model calculation $T_{i}=0$, we get for the final temperature $T_{f}=$ $\sqrt{(2 / \gamma)\left(E_{i}-E_{0}\right)}$, and, by recalling that $C_{V} / T=d S / d T$, we get the increase in entropy $\Delta S=\gamma T_{f}$. The fact that the energy $E_{i}-E_{0}$ is converted into heat that increases the temperature and entropy of the 2DEG allows us to identify $E_{i}-E_{0}$ as the maximum energy that could, in principle, be extracted from the motion in the $z$ direction to do work.

We can also use Eq. (13) to define a suitable "temperature" for nonequilibrium states. We observe that Eq. (13) can be cast in the form $\int_{0}^{\infty} d t\left[\frac{d E}{d t}+T(t) \frac{d S}{d t}\right]=0$, which simply states the global conservation of energy. This suggests that we define the nonequilibrium temperature of the system in such a way that the equation

$$
\frac{d E}{d t}=-T(t) \frac{d S}{d t}
$$

is satisfied at all $t$. The possibility of this definition stems from the fact that we can always follow a nonequilibrium thermodynamical transformation through a series of small quasiequilibrium transformations. Equation (14) is, in fact, a differential equation for the temperature that can be easily solved to give $T(t)=\sqrt{(2 / \gamma)\left[E_{i}-E(t)\right]}$ [we have used again $C_{V}(T)=\gamma T$ for a 2DEG] and, finally [see Fig. 1(c)],

$$
\Delta S(t)=\sqrt{2 \gamma\left[E_{i}-E(t)\right]} .
$$

In conclusion, we have shown that the time-dependent current-density-functional theory naturally opens the possibility of a first-principle description of relaxation in many-body systems. To show this, we have identified a quantity, the Kohn-Sham energy, that defines a "time arrow" in the evolution of the system by monotonically decreasing with time. We are also able to relate the energy dissipated to the increase in entropy and temperature. This thermodynamical approach allows us to identify the KohnSham energy with the maximum amount of work that can be extracted from the system.

We thank Carsten Ullrich for assistance with the numerical calculations and for many useful discussions and
Janmin Tao for a critical reading of the manuscript. We acknowledge financial support from NSF Grant No. DMR0313681 and the kind hospitality of the Scuola Normale Superiore in Pisa, where part of this work was completed.

[1] U. Weiss, Quantum Dissipative Systems (World Scientific, Singapore, 1999), 2nd ed.

[2] Introduction to Nanoscale Science and Technology, edited by M. Di Ventra, S. Evoy, and J.R. Heflin (Springer, Berlin, 2004).

[3] Atoms in Intense Laser Fields, edited by M. Gavrila (Academic, Boston, 1992).

[4] We assume that the system is large enough for the Poincaré recurrence time to be practically infinite.

[5] R. P. Feynman and F. L. Vernon, Ann. Phys. (N.Y.) 24, 118 (1963).

[6] P. Hohenberg and W. Kohn, Phys. Rev. 136, B864 (1964).

[7] W. Kohn and L. J. Sham, Phys. Rev. 140, A1133 (1965).

[8] E. K. U. Gross, J. F. Dobson, and M. Petersilka, in Density Functional Theory, edited by R.F. Nalewajski (Springer, Berlin, 1996), p. 81.

[9] R. van Leeuwen, Int. J. Mod. Phys. B 15, 1969 (2001).

[10] G. F. Giuliani and G. Vignale, Quantum Theory of the Electron Liquid (Cambridge University, Cambridge, England, 2005).

[11] For a different approach to dissipative systems in the framework of time-dependent density-functional theory, see K. Burke, R. Car, and R. Gebauer, Phys. Rev. Lett. 94, 146803 (2005).

[12] G. Vignale and W. Kohn, in Electronic Density Functional Theory: Recent Progress and New Directions, edited by J.F. Dobson, G. Vignale, and M.P. Das (Plenum, New York, 1996), p. 199.

[13] G. Vignale, C. A. Ullrich, and S. Conti, Phys. Rev. Lett. 79, 4878 (1997).

[14] We have $E_{0}[A, V]=\min _{\Psi}\left\{\left\langle\Psi\left|\hat{T}_{A}+\hat{V}\right| \Psi\right\rangle+E_{h x c}[n, A]\right\}=$ $\min _{\Psi}\left\{\left\langle\Psi\left|\hat{T}_{A}+\hat{V}+W\right| \Psi\right\rangle\right\}$

[15] G. Vignale, M. Rasolt, and D. J. W. Geldart, Phys. Rev. B 37, 2502 (1988).

[16] In a homogeneous electron liquid, the presence of a magnetic field tends to localize the electrons, thus deepening the $x c$ hole and, therefore, decreasing the $x c$ energy. If the inhomogeneity of the system is not so strong, we expect this to be more generally true.

[17] B. Simon, Phys. Rev. Lett. 36, 1083 (1976).

[18] For the system to get stuck in an excited state, the initial wave function should have certain symmetries that do not belong to the ground state. In experiments, it is difficult to prepare a many-particle system in such a state.

[19] H. O. Wijewardane and C. A. Ullrich, Phys. Rev. Lett. 95, 086401 (2005).

[20] A more accurate study of this system, including the frequency-dependence of the viscoelastic coefficients, has been recently presented in Ref. [19].

[21] L.D. Landau and E.M. Lifshitz, Statistical Physics (Pergamon, New York, 1980). 